\title{
Does anthropogenic introduction of guppy fish (Poecilia reticulata) impact faunal species diversity and abundance in natural aquatic habitats? A systematic review protocol
}

\author{
Misaki Sasanami ${ }^{1}$, John Hustedt ${ }^{2}$, Neal Alexander ${ }^{3}$, Olaf Horstick ${ }^{4}$ Leigh Bowman ${ }^{5}$, Jeffrey Hii ${ }^{6}$, \\ Pierre Echaubard ${ }^{7}$, Leo Braack ${ }^{8}$ and Hans J. Overgaard ${ }^{9,10^{*}}$ (D)
}

\begin{abstract}
Background: The guppy fish (Poecilia reticulata) is a tropical fish ancestrally linked to northern South America and the Caribbean. It is known to be very tolerant of and adaptable to new environments, and able to consume multiple food sources, including mosquito larvae. Consequently, guppies have been frequently introduced to non-native ecosystems to control mosquito populations, resulting in near-global distribution. Indeed, due to the increasing need for mosquito-borne disease control, guppy releases will likely continue, however there are concerns about potential adverse impacts on other species, biodiversity, and certain ecosystem functions. The most significant of these is local species extinction, and by extension, reduced biodiversity. Yet, the extent of these impacts has not been evaluated by scientific review. Accordingly, this study will examine and evaluate whether anthropogenic introduction of guppy fish (Poecilia reticulata) has impacts on faunal species diversity and abundance in natural aquatic habitats. The results of this review may have implications for environmental management and policy and inform ecosystem-based integrated vector management and public health policy.

Methods: Relevant scientific articles will be identified by searching electronic databases. Articles will be included if they report changes or differences, associated with guppy fish introduction, in at least one of these population parameters: (1) abundance of individuals in any species, (2) total abundance of individuals in all species present, (3) species richness, (4) species diversity, and (5) community evenness. Each article will be assessed by at least two independent reviewers against pre-defined inclusion/exclusion criteria. Snowballing reference lists of included articles will be conducted. At least two reviewers will critically appraise all included studies using the Collaboration for Environmental Evidence Critical Appraisal Tool (CEECAT) and any discrepancies will be resolved by discussion between the two or adjudication by a third author if agreement is not reached. Each study will also be subjected to data extraction against pre-defined qualitative and quantitative outcomes and results will be tabulated/presented in figures where appropriate. A meta-analysis will be carried out on outcome parameters with sufficient evidence.
\end{abstract}

Keywords: Integrated vector management, Larvivorous fish, Invasive species, Dengue, Ecosystem approach to health, Biodiversity, Alien fish

*Correspondence: hans.overgaard@nmbu.no

${ }^{9}$ Faculty of Science and Technology, Norwegian University of Life Sciences, P.O. Box 5003, 1432 Ås, Norway

Full list of author information is available at the end of the article

\section{Background}

The ancestral indigenous habitat for the guppy fish (Poecilia reticulata W. Peters, 1859) is northern South America and Central America [1], however it is now one of the 
most widely distributed tropical fish species in the world. It has been documented in 69 countries across each of the continents except Antarctica [2] (Fig. 1). Guppy fish can flourish in temperate areas such as Russia and England if water temperatures are higher than $10{ }^{\circ} \mathrm{C}$ [3], and are capable of colonizing many habitats including brackish waters [1]. Physiological characteristics, life history patterns and phenotypic plasticity enable guppies to evolve rapidly in response to environmental stressors such as predation risk; examples include altering age and size at maturity, size of offspring and reproduction frequency [1]. Female guppies are ovoviviparous (i.e., give birth to well-developed, independent offspring) [1], which affords improved survival rates when compared to oviparous species. In addition, females can store sperm from a single insemination to ensure several broods, which can lead to entirely new populations [4]. An omnivorous diet further ensures higher survival/reproduction rates, as food sources such as algae, organic detritus, diatoms, protozoa, zooplankton, fish parts and mosquito larvae are often widely available [5]. Their exceptional survivability, adaptability, fecundity and larvivorous diet has since made them favorable as a measure to control mosquito populations, which partly explains why guppies have been introduced to various locations throughout the world for more than a hundred years [1,5]. Indeed it has been suggested that mosquito control demand currently accounts for more than $40 \%$ of guppy introductions globally [2], and the increasing need for mosquito-borne disease control will likely continue to drive this practice.

Guppies are particularly used for dengue vector control $[6,7]$. Dengue is caused by the dengue virus (DENV) which is transmitted by the bite of Aedes mosquitoes. Dengue has become one of the leading causes of illness and death in the tropics and subtropics with a significant increase in incidence and an expansion into new geographic areas [8]. Due to the rise in dengue cases, and the current lack of available vaccines and therapeutics, there is an urgent need to identify more effective vector control methods, and guppy fish introduction to households and public places in peri-urban and rural areas is one alternative. In Cambodia, for example, the first largescale guppy introduction was carried out in 2007 bringing a sharp decline in the entomological indices (e.g., House, Breteau, and Container Indices) [9]. Subsequent studies have assessed the efficacy of using guppy fish in this context, and the benefits include: (1) high cultural acceptance in certain regions, such as in Southeast Asia; (2) the low cost of implementation; and (3) the potential for community-based actions, with or without state-led interventions [10-13], suggesting guppy introduction as an effective, well-accepted, and sustainable intervention. Thus, guppy introduction might play more important part in mosquito control, and be used even more widely in new geographical areas, or for other mosquito-borne disease control. Considering the potential risk for adverse environmental impacts by guppy release discussed in the next paragraph, the intervention might be more sustainable for container breeding vectors such as Aedes mosquitoes where these release into natural habitats are likely to be low.

However, the guppy is considered an invasive species $[14,15]$ that could adversely affect local ecosystems by reducing biodiversity and disrupting ecosystem functions [2, 16-19]. An experimental study conducted in Trinidadian streams found an increase in primary productivity as well as change in nitrogen fluxes following guppy introduction, suggesting effects on food web dynamics [20]. There was positive correlation between exotic poeciliid (fish in the Poeciliidae family) density and nitrogen level, and organic carbon yields in Hawaiian streams [21], which could be explained by the fact that fish convert organic nutrients into inorganic forms through excretion [22]. The dissolved form of nutrients is readily available for primary producers such as algae, potentially resulting in increased algal biomass [22]. It was also reported that native fish density was lower in poecilid-containing streams than in poeciliid-free ones [21] probably due to competition for limiting resources such as space and food [2, 21, 23]. Although the extent of ecosystem impact has not been independently reviewed, such impacts may be explained by the mechanisms shown in Fig. 2, with its ultimate, and most concerning, consequence being on faunal species diversity and abundance in natural aquatic habitats. Such impacts were observed with Gambusia, another larvivorous fish deployed worldwide for mosquito control, including reduced densities of aquatic invertebrates such as rotifers, crustaceans, backswimmers, water beetles, and odonatan larvae [24], which might also be affected by guppies. Indeed, documented impacts of guppies on faunal species include the local extinction of Halocaridina rubra in Hawaii [25] and lower densities of Trinidadian killfish (Rivulus hartii) [26]. From these observations, guppies might have the potential to affect not only other fish species of similar size or smaller or with similar niches, but also various invertebrate taxa including rotifers, crustaceans, and insects.

(See figure on next page.)

Fig. 1 Global distribution and origins of Poecilia reticulata including $\mathbf{A}$ their native distribution, $\mathbf{B}$ distribution as a result of introductions and $\mathbf{C}$ reported reasons behind introductions. Adapted from "How reproductive ecology contributes to the spread of a globally invasive fish" by Deacon AE, Ramnarine IW, Magurran AE, 2011, PLoS One, 6(9): e24416. Used under the Creative Commons Attribution License 


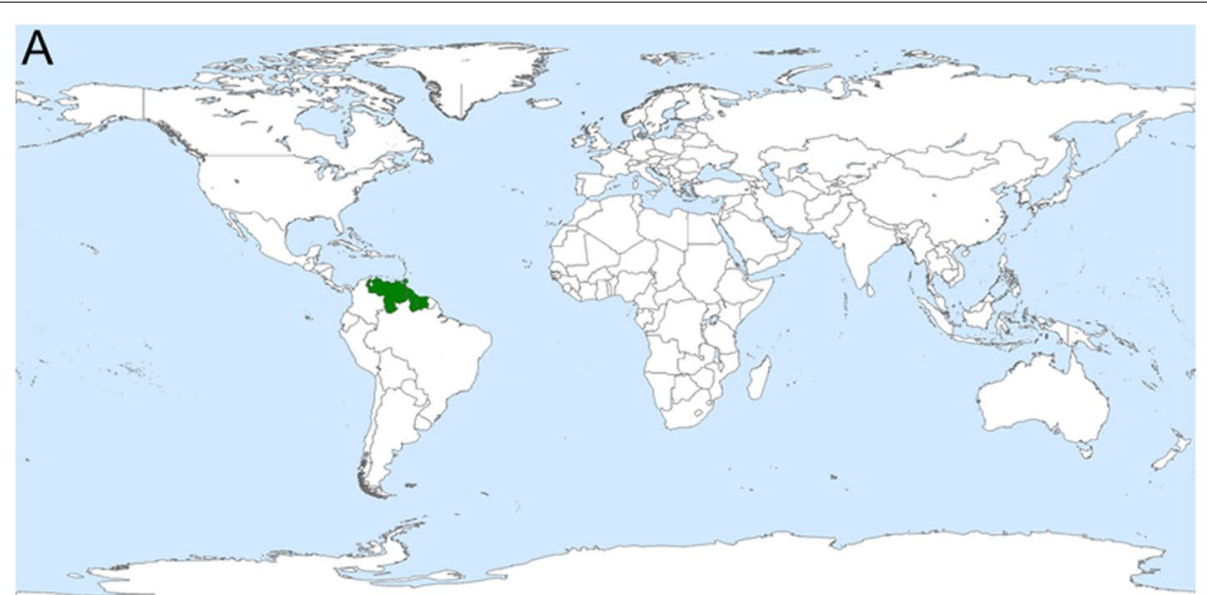

\section{Native range of Poecilia reticulata}
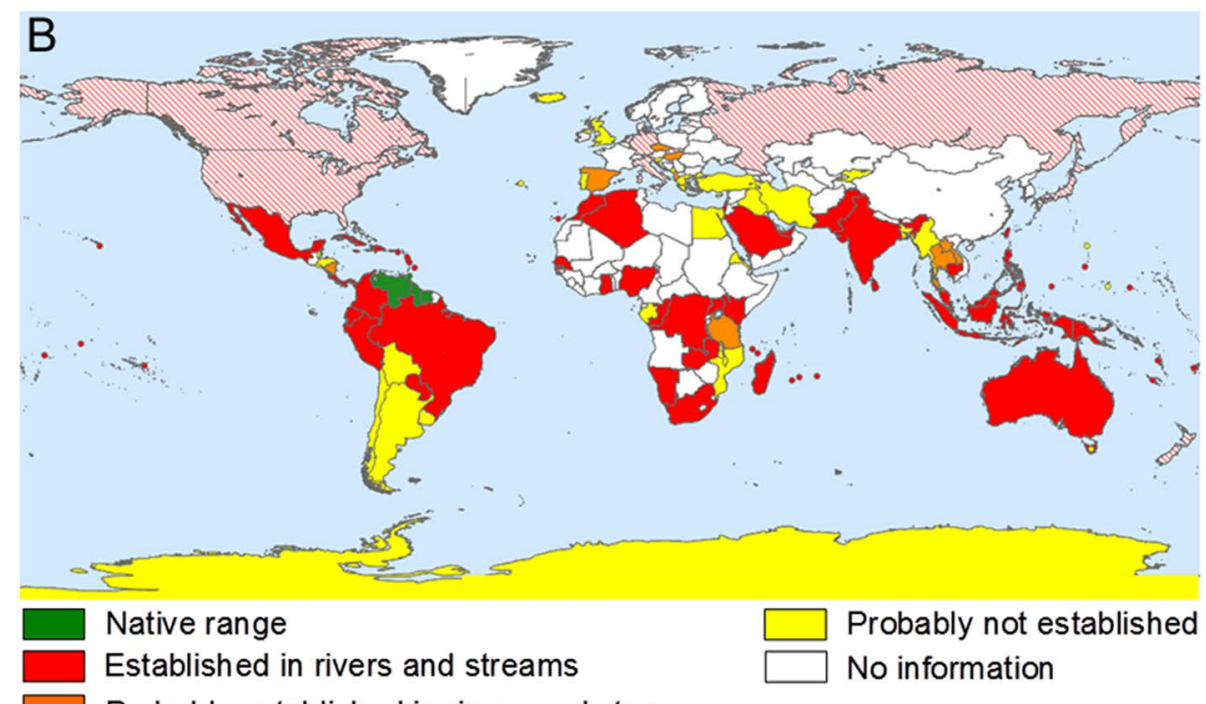

Probably established in rivers and streams

Established in geothermal/artificially heated waters

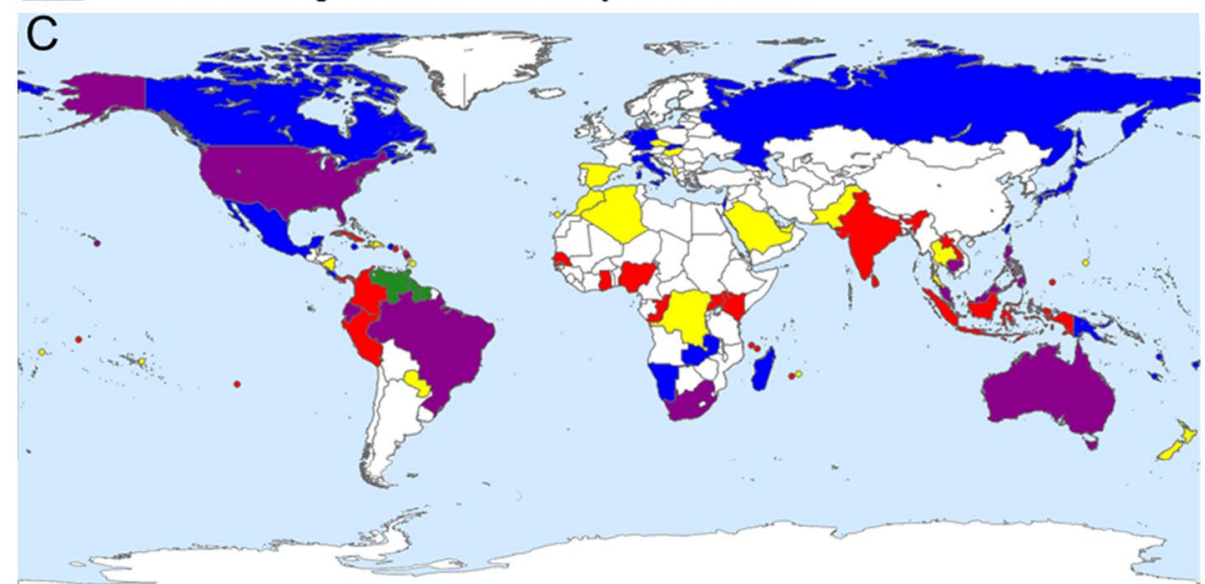




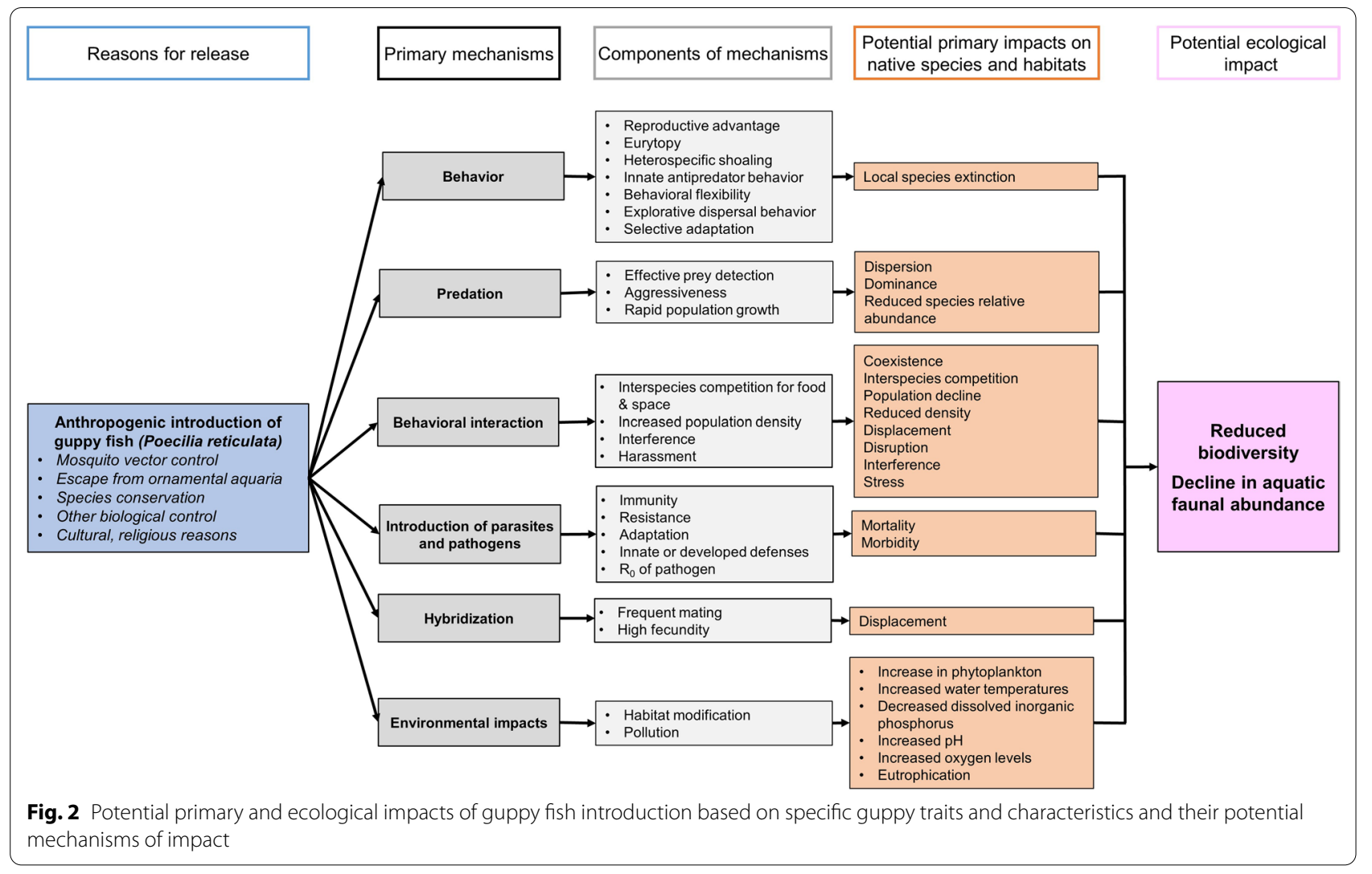

\section{Stakeholder engagement}

Following Land et al. [27] we implemented a four-step approach to stakeholder engagement including (1) stakeholder identification; (2) identification of relevant topics; (3) framing and prioritization of review questions; and (4) establishment of the specific scope of a review.

(1) Stakeholder identification

The initial stakeholder identification started with previous projects many of the authors were involved with [11, 13, 28, 29]. From 2010 to 2011, Dr. J. Hii and colleagues ran a randomized controlled trial in Cambodia and Laos which evaluated guppy fish distribution as one part of an Integrated Vector Management (IVM) strategy [13]. The project included both local and international stakeholders including funding agencies such as the Asian Development Bank and the World Health Organization (WHO). Subsequently, a larger cluster randomized trial was implemented in 2015-2016 using the lessons learned from the previous trial with support from the local government authorities, the WHO, UK Aid Direct (UKAID), and the German Corpora- tion for International Cooperation (GIZ) [11]. Due to the success of both trials, a follow-on project which expanded the target population to schools was implemented by Malaria Consortium together with the WHO Special Programme for Research and Training in Tropical Diseases (WHO-TDR) in 2018 [28]. However, all the previous projects had only evaluated the interventions with entomological indicators. An upcoming epidemiological trial run by the Norwegian University of Life Sciences (NMBU) and funded by the Norway Research Council will investigate the use of larvivorous fish as one part of an IVM strategy in Myanmar [30]. Many of these stakeholders from the vector control side including donors, international non-government agencies, universities, and governments were engaged and many of them from the ecological side were identified after results were published and the authors were contacted by groups interested in the topic $[16,17]$. In addition, Science magazine interviewed one of the authors to discuss the environmental aspects of guppy fish release for dengue vector control [31]. This led to a large variety of 
stakeholders being identified across several disciplines.

(2) Identification of relevant topics

The identification of topics, which were related to the chances of establishment of guppy populations in non-native environments and the impact of nonnative guppy populations on native flora and fauna, was decided after reading much of the literature and conferring with experts identified in step (1).

(3) Framing and prioritization of review questions

These topics were then discussed among the authors and refined to ensure we could answer the most pressing environmental concerns related to guppy fish introduction for dengue control.

(4) Establishment of the specific scope of a review After refining the topics, the authors framed and prioritized the review questions, assisted by the development of the search strategy. The establishment of the specific scope for the review and determination of the search strings were determined, debated, reformulated, and agreed upon by all authors.

\section{Objective of the review}

The objective of this study is to assess, by systematic review, the potential impact on the diversity and abundance of faunal species by anthropogenic introduction of guppy fish (Poecilia reticulata).

\section{Study question}

Does anthropogenic introduction of guppy fish (Poecilia reticulata) impact faunal species diversity and abundance in natural aquatic habitats?

\section{PICO components}

- Population: Aquatic habitats where guppy fish (Poecilia reticulata) were introduced anthropogenically

- Intervention/exposure: Anthropogenic introduction of guppy fish (Poecilia reticulata)

- Comparator: Before guppy introduction or contemporaneous 'control' aquatic habitats without presence of guppies.

- Outcome: Population parameters of aquatic fauna attributable to guppy fish introduction, namely abundance of individuals in any species (i.e., number of individuals, number of individuals per unit effort (or count per unit effort (CUE)), or pooled biomass); total abundance of individuals in all species present individuals (i.e., number of individuals, number of individuals per unit effort (or CUE), or pooled biomass); species richness (i.e., number of species); species diversity, measured by Shannon-Wiener or Simpson's indices; and community evenness, measured by Pielou's indices.

\section{Null Hypotheses}

1. Guppy fish (Poecilia reticulata) introductions in nonnative aquatic habitats do not affect single species abundance (e.g., number of individuals, number of individuals per unit effort (or CUE), or pooled biomass) under study.

2. There is no difference in community abundance (i.e., number of individuals, number of individuals per unit effort (or CUE), or pooled biomass) in all species present between aquatic habitats with and without introduced guppy fish (Poecilia reticulata).

3. There is no difference in species richness (i.e., number of species) between aquatic habitats with and without introduced guppy fish (Poecilia reticulata).

4. There is no difference in species diversity, measured by Shannon-Wiener or Simpson's indices between aquatic habitats with and without introduced guppy fish (Poecilia reticulata).

5. There is no difference in community evenness, measured by Pielou's indices between aquatic habitats with and without introduced guppy fish (Poecilia reticulata).

\section{Methods}

The reporting of this systematic review will be guided by the Collaboration for Environmental Evidence Guidelines and Standards for Evidence Synthesis in Environmental Management [32] and the Reporting Standards for Systematic Evidence Syntheses (ROSES) [see Additional file 1] [33].

\section{Searching for articles}

Articles will be first identified by searching academic electronic databases through the NMBU library. No restriction will be placed on the date of publication. In order to minimize the risk of publication bias, grey literature will also be searched through OpenGrey and GreenFILE, and snowballing will be used to identify additional articles from reference lists of included studies. In case the number of articles found is less than two after the screening process, we will use call-for-literature through social media such as Twitter or Facebook. Two articles is the minimum required number to perform a meta-analysis [34]. 


\section{Search languages}

Literature will be searched using English search terms, although screening will be conducted in English, Spanish, Portuguese, French and Japanese if any relevant literature is written in those languages.

\section{Search terms}

The search string was developed based on the Intervention 1 (I1), Intervention 2 (I2), and Outcome (O) PICO components (Table 1) in consultation with a librarian from NMBU. The Intervention 1 component contains terms about guppy fish, including scientific and vernacular names, synonyms, etc. Not search terms. The Intervention 2 component indicates reasons for why guppy interventions were applied, such as diseases, vectors, viruses, insect control, etc. The Outcome component denotes the selected outcome factors, Not search terms. Searches will be conducted in English, combining the three components using Boolean operators "AND" and "OR". Terms consisting of subject headings and/or text strings will be combined by "OR" within each component. Searches between components will be done by (I1 AND I2) OR (I1 AND O). The strings were adjusted to each database using the appropriate subject headings and text search [see Additional file 2]. Such adaptation will be reported in a supplementary table that shows the interface used, date of search, search terms with appropriate syntax, and the number of papers retrieved for each database in the final report.

\section{Sources}

The search will be applied to the following ten sources:

1. Web of Science

2. CAB Abstracts

3. Agricola

4. Embase

5. Biological Abstracts

6. MEDLINE
7. Scopus
8. GreenFILE
9. LILACS
10. OpenGrey

\section{Comprehensiveness check}

A comprehensiveness check of the search strategy was conducted [see Additional file 3] to see how many of the key papers can be obtained using all databases. Thirteen key papers were selected based on our knowledge and experience in the field and from some of the references used by Deacon et al. [2] and in other publications that were considered relevant. The check resulted in 92\% (12 of 13) of key papers being identified in at least one of the databases.

\section{Article screening and study eligibility criteria}

Search results will be exported to Covidence software (Melbourne, Australia. Available at www.covidence.org) in which duplicates will be identified and removed, and screening carried out.

\section{Screening process}

Using the eligibility criteria shown in Table 2, two reviewers will screen titles and abstracts and subsequently full text. Relevant papers written in English, Spanish, Portuguese, French, or Japanese will be screened. During the title and abstract screening in Covidence, each study will be categorized into "yes" or "no". The option "maybe" is also present in Covidence but this will be only used initially in case of doubt, pending a final decision. Studies will be included into "yes" only when they meet all the eligibility criteria. Studies with a conflict between the reviewers will be reviewed again by both reviewers. If an agreement cannot be reached, a third reviewer will make a final decision before proceeding to full text screening. The included articles will be subjected to full text screening where there are only two options "include" or

Table 1 General search terms (example taken from Web of Science)

\begin{tabular}{|c|c|}
\hline PICO component & Text search \\
\hline Intervention 1 & $\begin{array}{l}\text { (Poecilia or "rainbow fish" or (Girardinus "NEAR/1"(guppii or petersi or poecilioides* or poeciloides)) or Lebistes or lebister or "Lib- } \\
\text { istes poeciloides" or guppy or guppies or molly or mollies or gully or mosquitofish or "mosquito fish" or Haridichtys or "Acantho- } \\
\text { cephalus guppii" or "Acanthocephalus reticulatus" or Heterandia or millionfish or killifish) }\end{array}$ \\
\hline Intervention 2 & $\begin{array}{l}\text { Aedes or mosquito or ((Aedes or mosquito* or vector) "NEAR/1"(control or management)) or chikungunya or "chikun gunya" } \\
\text { or dengue or breakbone or dengue or zika or ((insect or mosquito or disease) "NEAR/1"vector*) or "disease transmission" or } \\
\text { "mosquito-borne disease*" or "aquarium dumping" }\end{array}$ \\
\hline Outcome & $\begin{array}{l}\text { biodiversity or "biological diversity" or (ecological "NEAR/1"(balance or effect* or system*)) or (species "NEAR/1"(diversity or rich- } \\
\text { ness or composition or evenness or abundance)) or ((community or population) "NEAR/1"(structure or abundance)) or (ecological } \\
\text { "NEAR/1"(impact or risk*)) }\end{array}$ \\
\hline
\end{tabular}




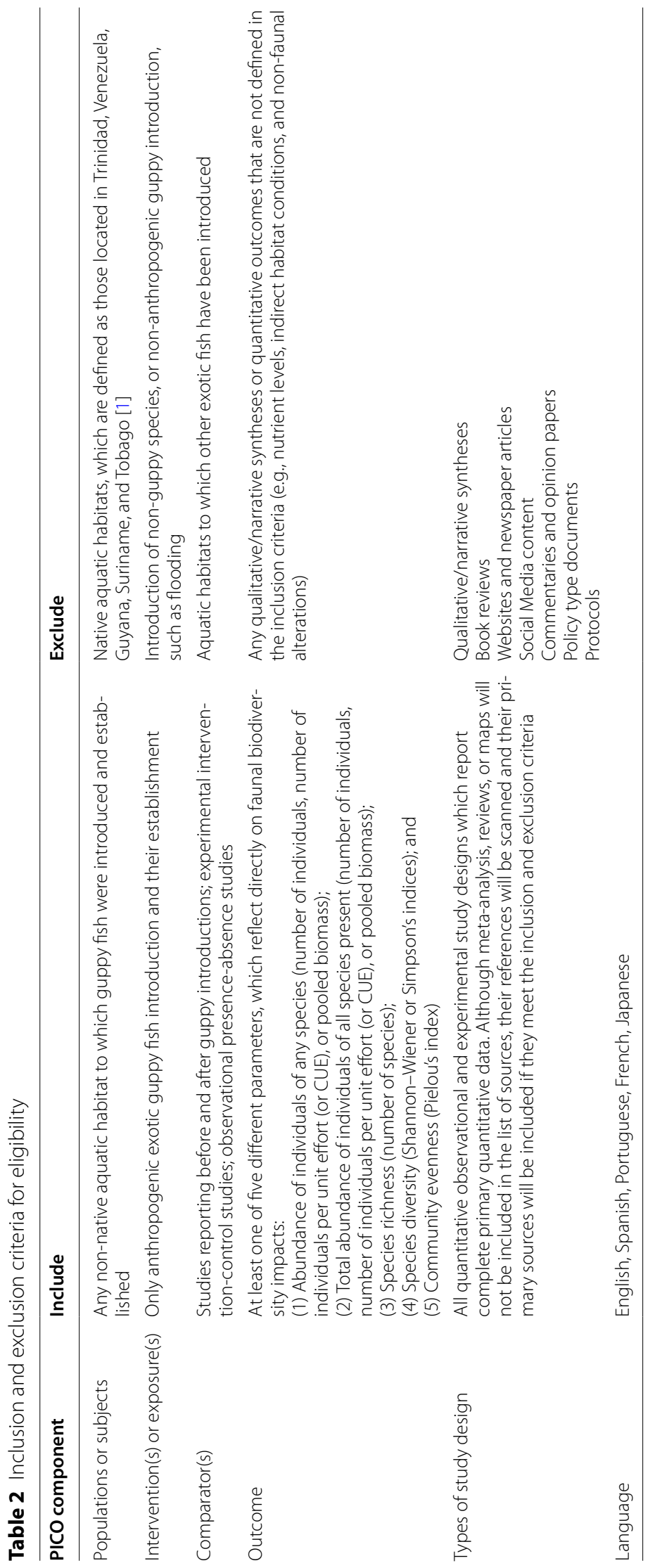


"exclude". The list of excluded studies at full-text screening will be provided in an additional file, stating reasons based on the PICO components according to the study eligibility criteria.

\section{Consistency checking}

Following title and abstract screening of all papers, the Kappa statistic will be calculated to evaluate consistency of inclusion between the two independent review authors using $10 \%$ of the articles written in English (which is the common language all reviewers are proficient in). A calculated Kappa value of $\geq 0.6$ signifies that consistency is ensured and only one reviewer will screen the remaining $90 \%$ of articles with the inclusion and exclusion criteria kept the same. If Kappa is $<0.6$ the inclusion and exclusion criteria will be revised, and two independent reviewers will screen all articles. At the full text screening, the decision on inclusion and exclusion will be made by at least two authors for all papers. Disagreements will be resolved in the same manner at the title and abstract screening.

\section{Eligibility criteria}

Long-term impacts, beyond one breeding or seasonal cycle, would be more ecologically relevant for biodiversity concerns. However, the availability of reported evidence on long-term impacts is uncertain, therefore we do not restrict impacts by time. In order to avoid circular argument and a voluminous additional portfolio of literature reporting on shifts in nutrient levels and indirect habitat conditions, we will confine inclusions to studies that reflect directly on faunal biodiversity impacts, i.e., measurable and significant shifts in species diversity and abundance. Changes in habitat conditions are therefore seen as an intermediate effect, while the actual impact of concern is the ultimate manifestation of habitat changes on species composition and relative or absolute abundance. We also confine inclusions to studies reporting the impacts on faunal species which are higher up the trophic chain than plants. Measuring faunal responses to guppy introduction therefore is closer to measuring ultimate impact of guppies on ecosystems as a whole.

\section{Study validity assessment}

Each paper that passes the full text screening will be subject to critical appraisal by at least two review authors. Study validity will be assessed based on the Collaboration for Environmental Evidence Critical Appraisal Tool (CEECAT) Version 0.2 (Prototype) [35] with potential confounders considered as those including seasonal effects, adverse climate events (drying out of the pool), and stochastic effects such as unusual nutritional input.
As a consistency check, all the reviewers who are responsible for critical appraisal will assess the study validity using $10 \%$ of the included studies written in English and the entries in the CEECAT and the data extraction sheet will be compared before conducting study validity assessment for all papers. Any discrepancies will be discussed, and the CEECAT and the data extraction sheet will be adjusted accordingly if that is considered necessary. In the formal assessment, any disagreements will be discussed between the reviewers, and in case agreement is not reached a third author will adjudicate them.

\section{Data coding and extraction strategy}

From all studies assessed in full and included in the investigation, data will be extracted to a data extraction sheet using Microsoft Excel with the following codebook categories [Additional file 4]:

1. Bibliographic information

2. Environment where the study was conducted

3. Impacts on faunal abundance, species richness and diversity, and community evenness

4. Other potential impacts

5. Potential effect modifiers/reasons for heterogeneity

6. Initial purpose of guppy introduction

Quantitative Outcome data will be extracted when stated explicitly in text or tables. In cases with unequal study units (e.g., two vs. one aquatic habitat with and without guppies), a simple (unweighted) average will be applied within each condition (e.g., to compare the average of two aquatic habitats versus one habitat). If there is ambiguity or opacity, we will submit a request to the corresponding author for data. If the requests are not answered we will try again, then try another author of the same paper. If not answered, we will only use the paper in the narrative synthesis but not in the quantitative analysis.

Prior to review in full, the data extraction sheet will be pilot tested on $10 \%$ of included studies while the reviewers will extract and code data independently. The entries in the sheet will be compared, and disagreements will be resolved by discussion between the two review authors. If no agreement can be reached, a third author will decide, and the data extraction will be revised accordingly. In the formal investigation, one review author will chart data and the second author will review the charted data with disagreements being solved by discussion between the two or judgement by a third author. If there is missing or unclear information in an article, the corresponding author of the article will be contacted for clarification via 
email. The data extraction sheet will be made available as an additional file for transparency.

\section{Potential effect modifiers/reasons for heterogeneity}

We have identified six key ecological effect modifiers that may affect the impact of exotic guppy introduction into a naive environment. These are (1) Habitat and niche diversity, (2) Diversity of resources, foodwebs and trophic levels, (3) Species richness, (4) Density dependence, (5) Behavioral change and (6) Ecological resilience. These factors are generally accepted ecological principles that apply to organisms occupying the same space that have overlapping resource requirements $[36,37]$.

1) Habitat and niche diversity

The more diverse a habitat is-for example in terms of availability of niches-the greater diversity of organisms it can host $[38,39]$ and this diversity of habitats, niches, and associated species is better capable of modulating a disturbance such as introduction of a foreign species [40, 41]. Habitats with many available niches provide hiding places for prey but also niches for many more different species of alternative prey organisms to colonize that guppy fish could exploit, thus ameliorating the impact of guppies on any one species.

2) Diversity of resources, foodwebs and trophic levels This is a subset of habitat diversity and follows the same logic as habitat diversity but relates specifically to diversity of food options available. Introduction of guppies into aquatic habitats with poor nutrient and food availability might be more dramatic and severe than in more diverse environments. Availability of alternative food sources may deflect predator pressure by guppies.

3) Species richness

The introduction of guppies in aquatic habitats with low species richness would typically be more dramatic and severe than in species-rich environments [42]. Potential negative impact of guppies will in such cases be distributed across the diversity of species present or regulated via functions arising by the diverse array of species present.

4) Density dependence

This means that population growth is controlled by its density (the severity of an effect depends on certain density thresholds). As the numbers of individuals in a species increase in a restricted habitat the species will be increasingly regulated by various factors, such as disease, competition, predation, and even density-induced hormonal reproductive inhibition. Outcome of guppy introduction therefore depends on the population dynamics and the nature and characteristics of species present in the introduced habitat.

5) Behavioral change

Behavioral change can be a potential impact-modifying response to guppy fish introduction. For example, some fish species may change normal behavior by increasing reproductive output to compensate for predation impact or adopt increased tendencies to frequent pool-bottom to avoid mingling with guppies that tend to be more active at the surface. Other species may tend to clump or cluster to present a larger and more confusing target that makes individual prey less susceptible to predation.

6) Ecological resilience

This is the ability of an ecosystem to resist, adapt and recover from external disturbances. General resilience is a function of environmental characteristics and ecosystem attributes [43-45] and, therefore, contain most of the modifiers discussed above, i.e., a more diverse ecosystem is more capable of absorbing shocks and bouncing back to its original functioning state without shifting regime. Some species are narrowly specialized and are specific in food requirements, so that if guppy fish exploit an overlapping resource (such as mosquito larvae) that they specialize in, it would impact more severely than on a "generalist" species that is an omnivore with multiple food tolerance. Some species are better able to adapt to habitat disturbances and respond to ecological pressures than others, and therefore more adept at resisting guppy fish challenge.

We have identified these effect modifiers which might influence the outcomes in studies on guppy introduction. We will extract information from each included article if such information exists and assess whether they might have affected review outcomes.

\section{Data synthesis and presentation}

A summary of findings will be narratively synthesized and presented in a table as well as text. We are planning a meta-analysis of outcomes and a publication bias assessment, although this depends on the availability and comparability of outcome data and study design and final decision can therefore only be made when data have been extracted. At this stage, we plan to calculate means and standard deviations (SD) for outcome measures, or extract them from published papers (Table 2), and we may analyze either the values on the original scale or use either Hedges' $g$ [46] or log-transformed values [47]. We intend to analyze the results using standard randomeffects models [48], within a frequentist framework without a prior distribution for the effect size. As a tool for 
visualizations and meta-analysis, $\mathrm{R}$ will be used. In the meta-analysis, studies with low or very low quality will be excluded, whilst they will still be used in the narrative synthesis. The effect of such exclusion will be evaluated by conducting sensitivity analysis if the meta-analysis is performed. Subgroup analysis might be carried out according to taxonomic levels of non-guppy fauna. The possible influence of publication bias might be explored using funnel plots if the number of studies is ten or more in the meta-analysis [49]. Also, the protocols of included studies will be examined to see if the outcomes stated in the protocol were subsequently published [50]. Studies with incomplete datasets will be excluded in the investigation (Table 2). Information on effect modifiers will be extracted from each article if they have been analyzed or mentioned [see Additional file 4]. Modifiers will not be analyzed quantitatively but will be included in the narrative analysis and discussed. We will include a discussion on implications for environmental management and policy and on how results can inform ecosystem-based IVM and public health policy.

\section{Supplementary Information}

The online version contains supplementary material available at https://doi. org/10.1186/s13750-021-00248-6.

Additional file 1. ROSES for Systematic Review Protocols. Version 1.0. ROSES form for this systematic review.

Additional file 2. Literature search specific for each database. Description of selected databases with adjusted search strings and search results and brief description about each database.

Additional file 3. Comprehensiveness check for search strategy. Selected databases with search results and list of key papers.

Additional file 4. Codebook for data extraction. Codebook for data extraction sheet.

\section{Acknowledgements}

We thank Johanne Longva, librarian at NMBU library, for advise on development of search strings.

\section{Authors' contributions}

MS and JHu drafted the protocol with input from all the other authors. All authors read and approved the final manuscript.

\section{Funding}

$\mathrm{HJO}, \mathrm{JHu}$, and JB are partly supported by the MY-SCHOOL project funded by the Research Council of Norway (RCN no. 285188). NA receives salary support from the MRC and the UK Department for International Development (DFID) under the MRC/DFID Concordat agreement, which is also part of the EDCTP2 programme supported by the European Union, grant reference: MR/ R010161/1. However, no funds were directly allocated for work on this review.

\section{Availability of data and materials}

The datasets used and/or analyzed during the current study will be published along with the systematic review manuscript.

\section{Declarations}

Ethics approval and consent to participate Not applicable.

\section{Consent for publication}

Not applicable.

\section{Competing interests}

The authors declare that they have no competing interests.

\section{Author details}

${ }^{1}$ Kyoto University School of Public Health, Yoshida-Konoe, Sakyo, Kyoto 606-8601, Japan. ${ }^{2}$ Health Forefront Organization, \#5, Street 282, Phnom Penh BKK1, Cambodia. ${ }^{3}$ MRC International Statistics and Epidemiology Group, London School of Hygiene \& Tropical Medicine, Keppel Street, London WC1E 7HT, UK. ${ }^{4}$ Heidelberg Institute of Global Health, University of Heidelberg, Im Neuenheimer Feld 365, 69120 Heidelberg, Germany. ${ }^{5}$ Faculty of Medicine, School of Public Health, Imperial College London, Norfolk Place, London W2 1PG, UK. ${ }^{6}$ School of Public Health, Tropical Medicine and Rehabilitation Sciences, James Cook University, 1 James Cook Dr, Douglas, QLD 4811, Australia. ${ }^{7}$ SOAS University of London, Thornhaugh Street, London WC1H OXG, UK. ${ }^{8}$ Malaria Consortium, Faculty of Tropical Medicine, Mahidol University, 420/6 Rajavithi Rd, Ratchathewi, Bangkok 10400, Thailand. ${ }^{9}$ Faculty of Science and Technology, Norwegian University of Life Sciences, P.O. Box 5003, 1432 Ås, Norway. ${ }^{10}$ Department of Microbiology \& Tropical Disease Research Center, Faculty of Medicine, Khon Kaen University, Khon Kaen, Thailand.

Received: 1 February 2021 Accepted: 14 November 2021

Published online: 04 December 2021

\section{References}

1. Magurran AE. Evolutionary ecology: the Trinidadian guppy. Press: Oxford Univ; 2005.

2. Deacon AE, Ramnarine IW, Magurran AE. How reproductive ecology contributes to the spread of a globally invasive fish. PLOS ONE. 2011;6:e24416.

3. Use of fish for mosquito control [Internet]. World Health Organization; 2003. https://applications.emro.who.int/dsaf/dsa205.pdf?ua=1

4. Carvalho GR, Shaw PW, Hauser L, Seghers BH, Magurran AE. Artificial introductions, evolutionary change and population differentiation in Trinidadian guppies (Poecilia reticulata: Poeciliidae). Biol J Linn Soc. 1996;57:219-34.

5. Lawal MO, Edokpayi CA, Osibona AO. Food and feeding habits of the Guppy, Poecilia reticulata, from drainage canal systems in Lagos, Southwestern Nigeria. West African J Appl Ecol. 2012;20:1-9.

6. Seng CM, Setha T, Nealon J, Socheat D, Chantha N, Nathan MB, et al. Community-based use of the larvivorous fish Poecilia reticulata to control the dengue vector Aedes aegypti in domestic water storage containers in rural Cambodia. J Vector Ecol [Internet]. C.M. Seng, WHO Cambodia 177-179, Pasteur Street 51, Phnom Penh, Cambodia: Society for Vector Ecology (1966 Compton Ave., Corona CA 91719, United States): 2008;33:139-44. http://www.sove.org/JournalPDF/June2008/16-Senge tal07-92.pdf

7. Lardeux F, Rivière F, Séchan Y, Loncke S. Control of the Aedes vectors of the dengue viruses and Wuchereria bancrofti: the French Polynesian experience. Ann Trop Med Parasitol [Internet]. F. Lardeux, Centre IRD (Institut de Recherche pour le Developpement, C.P. 9214, La Paz, Bolivia.; 2002;96 Suppl 2:S105-116. http://ovidsp.ovid.com/ovidweb.cgi?T=JS\& $\mathrm{PAGE}=$ reference $\& \mathrm{D}=$ emed7 $\& \mathrm{NEWS}=\mathrm{N} \& A \mathrm{~N}=36496106$

8. World Health Organization. Dengue guidelines for diagnosis, treatment, prevention and control. World Heal Organ. 2009.

9. Partnership IF. Regional Public Goods for Health : Combating Dengue in ASEAN ( Financed by the Regional Cooperation and Integration Fund under the Regional Cooperation and Integration Financing Partnership Facility ). 2009.

10. Echaubard P, Thy C, Sokha S, Srun S, Nieto-Sanchez C, Grietens KP, et al. Fostering social innovation and building adaptive capacity for dengue 
control in Cambodia: A case study. Infect Dis Poverty [Internet]. P. Echaubard, SOAS University London, Thornhaugh Street, London WC1H 0XG, United Kingdom. E-mail: pe5@soas.ac.uk, C. Thy, Malaria Consortium, Phnom Penh, Cambodia. E-mail: cheacheathy@gmail.com: BioMed Central Ltd (United Kingdom. E-mail: info@biomedcentral.com); 2020;9:126. http://www.idpjournal.com/

11. Hustedt JC, Doum D, Keo V, Ly S, Sam B, Chan V, et al. Field efficacy of guppies and pyriproxyfen (Sumilarv ${ }^{\circledR} 2 \mathrm{MR}$ ) combined with community engagement on dengue vectors in Cambodia: a randomized controlled trial. bioRxiv [Internet]. 2020. https://www.biorxiv.org/content/https://doi. org/10.1101/2020.05.15.097782v1

12. Shafique M, Lopes S, Doum D, Keo V, Sokha L, Sam BL, et al. Implementation of guppy fish (Poecilia reticulata), and a novel larvicide (Pyriproxyfen) product (Sumilarv 2MR) for dengue control in Cambodia: A qualitative study of acceptability, sustainability and community engagement. PLoS Negl Trop Dis [Internet]. M. Shafique, Technical Department, Malaria Consortium, Phnom Penh, Cambodia. E-mail: muhammad. shafique2002@gmail.com: Public Library of Science (E-mail: plos@ plos.org); 2019;13:e0007907. https://journals.plos.org/plosntds/article/ file?id=https://doi.org/10.1371/journal.pntd.0007907\&type=printable

13. Asian Developmental Bank. Managing regional public goods for health: community-based dengue vector control. Asian Dev. Bank World Heal. Organ. 2013.

14. Animalia K, Chordata P, Actinopterygii C, Cyprinodontiformes FP. FULL ACCOUNT FOR: Poecilia reticulata Poecilia reticulata. 2017;1-5.

15. CABI. Invasive Species Compendium [Internet]. https://www.cabi.org/isc/ datasheet/68208\#tosummaryOfInvasiveness. Accessed 4 July 2021.

16. El-Sabaawi RW, Frauendorf TC, Marques PS, Mackenzie RA, Manna LR, Mazzoni R, et al. Biodiversity and ecosystem risks arising from using guppies to control mosquitoes. Biol Lett. 2016;12:2016-9.

17. Azevedo-Santos VM, Vitule JRS, García-Berthou E, Pelicice FM, Simberloff D. Misguided strategy for mosquito control. Science (80-). 2016. https:// doi.org/10.1126/science.351.6274.675.

18. Deacon AE, Magurran AE. How behaviour contributes to the success of an invasive Poeciliid Fish: the Trinidadian Guppy (Poecilia reticulata) as a model species. Biol Invasions Anim Behav. 2016. https://doi.org/10.1017/ CBO9781139939492.016.

19. Bassar RD, Marshall MC, López-Sepulcre A, Zandonà E, Auer SK, Travis J, et al. Local adaptation in Trinidadian guppies alters ecosystem processes. Proc Natl Acad Sci U S A. 2010. https://doi.org/10.1073/pnas.0908023107.

20. Collins SM, Thomas SA, Heatherly IIT, MacNeill KL, Leduc AOHC, LópezSepulcre A, et al. Fish introductions and light modulate food web fluxes in tropical streams: a whole-ecosystem experimental approach. Ecology [Internet]. United States; 2016;97:3154-66. http://ovidsp.ovid.com/ovidw eb.cgi?T $=J S \& P A G E=$ reference $\& D=$ emed $17 \& N E W S=N \& A N=616858883$

21. Holitzki TM, MacKenzie RA, Wiegner TN, McDermid KJ. Differences in ecological structure, function, and native species abundance between native and invaded Hawaiian streams. Ecol Appl. 2013;23:1367-83.

22. Vanni MJ. Nutrient cycling by animals in freshwater ecosystems. Annu Rev Ecol Syst. 2002;33:341-70

23. Valero A, Macías Garcia C, Magurran AE. Heterospecific harassment of native endangered fishes by invasive guppies in Mexico. Biol Lett. 2008:4:149-52.

24. Pyke GH. Plague minnow or mosquito fish? A review of the biology and impacts of introduced Gambusia species. Annu Rev Ecol Evol Syst. 2008;39:171-91

25. Brock, R. E., \& Kam AK. Biological and water quality characteristics of anchialine resources in Kaloko-Honokohau National Historical Park. Geographical. 1997;96822.

26. Walsh MR, Fraser DF, Bassar RD, Reznick DN. The direct and indirect effects of guppies: Implications for life-history evolution in Rivulus hartii. Funct Ecol. 2011. https://doi.org/10.1111/j.1365-2435.2010.01786.x.

27. Land M, Macura B, Bernes C, Johansson S. A fve-step approach for stakeholder engagement in prioritisation and planning of environmental evidence syntheses. Environ Evid. 2017. https://doi.org/10.1186/ s13750-017-0104-0.

28. World Health Organization. Schoolchildren in Cambodia fight dengue using guppy fish [Internet]. 2019. https://www.who.int/tdr/news/2019/ schoolchildren-in-Cambodia-fight-dengue/en/. Accessed 5 May 2021.
29. Norwegian University of Life Sciences. MY-SCHOOOL_fighting dengue in Myanmar [Internet]. https://www.nmbu.no/en/projects/node/41250 Accessed 5 May 2021.

30. Research Council of Norway. School and community-based studentdriven dengue vector control and monitoring in Myanmar: a cluster randomized controlled trial. Project no. 285188. [Internet]. https://prosj ektbanken.forskningsradet.no/en

31. Servick K. Ecologists raise alarm over releases of mosquito-killing guppies. Science (80-). 2016. https://doi.org/10.1126/science.aal0304.

32. Pullin A, Frampton G, Livoreil B, Petrokofsky G. Guidelines and standards for evidence synthesis in environmental management. Version 5.0. Collab Environ Evid. 2018

33. Haddaway N, Macura B, Whaley P, Pullin A. ROSES for systematic review protocols. Version. 2017.

34. Ryan R. Cochrane Consumers and Communication Review Group reviews: Mata-analysis. Cochrane Consum Commun Rev Gr [Internet]. 2016;2016:1-6. http://cccrg.cochrane.org

35. CEE Critical Appraisal Tool [Internet]. Collab. Environ. Evid. 2021. https:// environmentalevidence.org/cee-critical-appraisal-tool/

36. Zaret TM, Rand AS. Competition in tropical stream fishes: support for the competitive exclusion principle. Ecology. 1971;52:336-42.

37. Muthukrishnan R, Hansel-Welch N, Larkin DJ. Environmental filtering and competitive exclusion drive biodiversity-invasibility relationships in shallow lake plant communities. J Ecol. 2018. https://doi.org/10.1111/ 1365-2745.12963.

38. Kallimanis AS, Mazaris AD, Tzanopoulos J, Halley JM, Pantis JD, Sgardelis SP. How does habitat diversity affect the species-area relationship? Glob Ecol Biogeogr. 2008. https://doi.org/10.1111/j.1466-8238.2008.00393.x.

39. Davies KF, Chesson P, Harrison S, Inouye BD, Melbourne BA, Rice KJ. Spatial heterogeneity explains the scale dependence of the native-exotic diversity relationship. Ecology. 2005;86:1602-10.

40. Linder PH, Bykova O, Dyke J, Etienne RS, Hickler T, Kühn I, et al. Biotic modifiers, environmental modulation and species distribution models. J Biogeogr. 2012. https://doi.org/10.1111/j.1365-2699.2012.02705.x.

41. Liso S, Gjelland K, Amundsen PA. Resource partitioning between pelagic coregonids in a subarctic watercourse following a biological invasion. J Ichthyol. 2013. https://doi.org/10.1134/S0032945213010074.

42. Michalet R, Brooker RW, Cavieres LA, Kikvidze Z, Lortie CJ, Pugnaire Fl, et al. Do biotic interactions shape both sides of the humped-back model of species richness in plant communities? Ecol Lett. 2006;9:767-73.

43. Gunderson LH. Ecological resilience-in theory and application. Annu Rev Ecol Syst. 2000;31:425-39.

44. Holling CS. Resilience and stability of ecological systems. Futur Nat Doc Glob Chang. 2013;4:23.

45. Chambers JC, Allen CR, Cushman SA. Operationalizing ecological resilience concepts for managing species and ecosystems at risk. Front Ecol Evol. 2019. https://doi.org/10.3389/fevo.2019.00241.

46. Hedges LV. Distribution theory for Glass's estimator of effect size and related estimators. J Educ Stat. 1981;6:107-28.

47. Higgins JPT, White IR, Anzures-Cabrera J. Meta-analysis of skewed data: combining results reported on log-transformed or raw scales. Stat Med. 2008;27:6072-92.

48. Borenstein M, Hedges LV, Higgins JPT, Rothstein HR. A basic introduction to fixed-effect and random-effects models for meta-analysis. Res Synth Methods. 2010;1:97-111.

49. Sterne JAC, Sutton AJ, loannidis JPA, Terrin N, Jones DR, Lau J, et al. Recommendations for examining and interpreting funnel plot asymmetry in meta-analyses of randomised controlled trials. BMJ. 2011;343:1-8.

50. Ayorinde AA, Williams I, Mannion R, Song F, Skrybant M, Lilford RJ, et al. Assessment of publication bias and outcome reporting bias in systematic reviews of health services and delivery research: a meta-epidemiological study. PLoS ONE. 2020;15:1-17. https://doi.org/10.1371/journal.pone. 0227580 .

\section{Publisher's Note}

Springer Nature remains neutral with regard to jurisdictional claims in published maps and institutional affiliations. 